\title{
General Discussion to papers of Dr E. McClemont and Dr Frankel et al.
}

Chairman: MR P. HARRIS (G.B.). Thank you very much indeed Dr Matthias for a most interesting and fascinating study. Now these two papers are open for discussion.

Sir LUDWIg GutTMANN (G.B.). These interesting papers have opened a new field of research in spinal cord injuries. I am particularly interested in this subject as I had for quite a number of years the opportunity to treat schizophrenics with insulin shock therapy and found profound changes of the cardiovascular system during and after hypoglycaemia. Even partial heart block was observed at the height of hypoglycaemia. However, following intravenous glucose injections these dramatic functional changes of the heart disappear swiftly. Dr McClemont did not mention details of changes of the autonomic system during her experiments but on the other hand she reported, if I understand correctly, one of the cases had an epileptic fit, which usually occurs at a certain stage of insulin shock. Therefore, I would like to ask her was it the only case of epileptic attack you had and, secondly, have you done running electrocardiograms and other clinical tests?

With regards to Mathias' and Frankel's paper, you have mentioned some of the cardiovascular changes but I am surprised that there are not more changes of catecholamines. This is a research which should be really continued and I would like to know whether you have made a series of electrocardiograms from the start and throughout the experiment.

Mr HARRIS. Dr McClemont, I think it would be appropriate if you spoke now.

Dr McClemont (G.B). I'm sorry, Sir Ludwig, if my Scottish accent has misled you but none of my patients did have an epileptic fit, and we did continue with ECG recording in all patients. We had continuous ECGs monitoring all patients.

DR H. Frankel (G.B.). These studies were done on volunteers and were very closely monitored both with continuous electrocardiogram and very frequent glucose estimations, and if we had run into the level of hypoglycaemia that you used to give schizophrenics we would have aborted the experiment before we reached that stage. I presume that in your patients they were suffering from hypokalaemia at the time they had their problems, but it is of practical importance. Our studies were done with the patients lying down and we induced hypotension, a relative hypotension in patients who in any case we know to be very hypotensive when in the sitting-up position, so we can deduce that hypoglycaemia in a tetraplegic will be extremely dangerous, because there will be no warning of it and it will be added to extreme hypotension both of which may damage brain tissues. It does tell us that at least in insulin-taking tetraplegics, of which we fortunately have a very small number, it is a very hazardous situation.

DR M. Agerholm (G.B.). I am now going to risk losing some very good friends, but I would like to make my protest against what I consider experimenting on people who are virtually laboratory specimens. I know this is unpopular to say, we have all known about the low blood pressures and the low blood sugars. I know people want to explain it. This protest that I just feel driven to make is partly because when I had poliomyelitis patients, I used to find them volunteering for all sorts of investigations. I don't think that a volunteer who is himself a patient is always a suitable subject for this kind of work. If doctors themselves have a cervical injury I think they are entitled to give themselves like this but I'm terribly sorry, I do regard this as something that is in my book unethical. I accept that it isn't in others. I just feel I must make this protest.

MR P. HARRIS. Dr Agerholm, I assure your precedent, and my comment here I have query ethics. I think this goes through all of our work as doctors whether we are dealing in the field of paraplegia or what have you, and certainly in this country it is much more difficult to obtain a certificate from the Home Office for animal experimentation than in humans. But this is a good subject and it may be a good topic some 
day for one of our meetings that I have this down here and that our patients are paraplegics, they are sitting birds, and volunteers. I go along with you, but I wouldn't like to pursue this further. I think the point has been made and any speakers can speak on this.

Petchacombie (U.S.A.). I respectfully disagree with you. I consider that we have to learn a little more of the physiology of these patients. We are all interested in how these patients interrelate to certain aspects of stress and insulin hypoglycaemia is one. Also it was mentioned that this proposal has some clinical application, a minority of patients suffer from hypoglycaemia and they have to be warned of the fact that they lack this alarm response. I really think that these patients, although they volunteered for this, are doing themselves a service because they are learning about their own disease and also helping us to learn about the inter-relation too during the stress. I would like to congratulate the two presenters. My own experience is quite similar, I don't have the data to show you but we have done four patients with high-level tetraplegia, actually we also demonstrated with a different value assay, that actually the levels of epinephria norapenephrin are quite low in a tetraplegic patient. We have measured cortisone levels and haemocrit levels and these levels are quite normal.

Dr H. J. Hachen (Switzerland). I have a question to both Dr McClemont and Dr Frankel, regarding not hypo- but hyperglycaemia. Have you noticed in patients who have had hyperglycaemia what were the general reactions you mentioned in hypoglycaemia? This is interesting first because in the acute stage several of our tetraplegic patients in intensive care received 10 or even 20 per cent glucose and this must be compensated three to four times daily, and there is danger that these patients may have peaks both high and low glucose and actually when I remember now when I think back to these cases they have been surprisingly little or hardly ever been annoyed by any general phenomena due to extreme values of hypo- or hyperglycaemia.

Mr HARRIS. I think we might have one more question and give the two authors chance to reply. One more question?

DR J. Vernon (G.B.). I'd just like to mention one patient with diabetes on my list, perhaps a very important practical point. He came for an IVP examination for an incomplete cervical patient who used to walk. He had been told he must not have any breakfast before the IVP so he had no insulin. He had the hangover from the previous day's insulin. He was rather sleepy, he was never very bright, and before he went off we found his blood glucose was down to $40 \mathrm{mgms}$. Now this is the sort of patient you might get if they come up for an IVP examination not taking their insulin. This sort of thing is forgotten. I just mention this one case but he might have died had we not spotted it in time.

MR P. HARRIS. Thank you very much for this very practical point. Now could we ask Dr McClemont first if she wishes to answer any points.

Dr McClemont (G.B.). I don't. I think Dr Mathias is probably more metabolically able than I am, but we haven't looked at hyperglycaemia at all in any of our patients.

Mr P. HARRIS. Dr Frankel ?

DR H. Frankel (G.B.). First of all regarding Dr Hachen's question. I am not really familiar with any marked symptoms of hyperglycaemia in normal people except visual disturbances if it's chronic. I don't know what he thinks we should be missing, but I presume we would miss it if there were any. Regarding the ethical question, this naturally has exercised my mind quite a lot in much of the research we do and I did present a paper to this Society on Ethical Considerations in Treatment and Research in our field a number of years ago, and I do take an interest in it. I'm on our ethical committee, I'm the chairman of it for the District, although naturally I don't adjudicate on my own application. I can assure Dr Agerholm that these were as genuine a volunteer as you can get and that we had far more refusals to cooperate in the studies than we had acceptances. A number of the patients also have hypotension or other problems which are investigated concurrently with the same apparatus which is then used for this study. I appreciate that one cannot have an absolute guarantee of total safety of the procedure 
and that we cannot be absolutely certain that the patients are genuine volunteers. However, taking all this into consideration I did feel it right to proceed with the study and these are the results.

MR P. HARRIS. I would like to thank the speakers and those who took part in the discussions for all they have contributed. I'd like to comment on the very high quality I think throughout the meetings not only on the papers but on the slides. 